\title{
Considerations regarding drug interactions at smokers
}

\author{
D Cîrţînă ${ }^{1}, V$ Nănescu ${ }^{1}$ and $R$ Mecu ${ }^{1}$ \\ 1“'Constantin Brâncusi”" University of Tg-Jiu, Faculty of Medical and Behavioral Sciences, No. \\ 4, Tineretului St., Tg-Jiu, Romania \\ E-mail: $\underline{\text { danielacirtina@gmail.com }}$
}

\begin{abstract}
This paper presents the way in which it can affect cigarette smoke, through its chemical composition, the action of a drug and its therapeutic efficacy in the treatment of smokers patients. This interaction can reduce the effect of drugs, the result of drug therapy can undergo severe changes in smokers. The most clinically important drug interactions with smoking may be depending on their effect: pharmacokinetic, pharmacodynamic, and carrier interactions. Since tobacco use is the most important health risk, which can be avoided, drugdrug interactions are identified. an important responsibility and task of the doctor and pharmacist.
\end{abstract}

Keywords: drug interactions, cigarette smoke, smoker, nicotine

\section{Introduction}

Smoking is one of the most harmful habits of the population, almost a widespread practice nowadays, which does not find any basis in any necessity and whose extension can hardly be explained historically, psychologically and afterwards. criteria. This defect is the most important cause of premature death in the European Union, causing annually the death of over 700.000 people, the death occurring in most cases about 14 years earlier.

The onset of smoking occurs in a significant proportion of cases in people who ignore or underestimate the effects of smoking. Psychological studies have highlighted regarding the initiation of smoking the role of the tendency to assert personality, especially in the periods when the individual poses such problems, ie at puberty. and adolescent.

In cigarette smoke there are over 4000 substances, over 65 of them being cancerous, among the most important being nicotine, polycyclic aromatic hydrocarbons, carbon oxide, acetaldehyde, acetonitrile, hydrocyanic acid, ammonia, amines, nitrosamines, aldehydes, ketones, alcohols, phenols, metals. Thus, smoking can affect both the pharmacokinetics and pharmacodynamics of drugs in therapeutic doses in the case of smokers having to be adjusted.

Tobacco use is most often associated with various forms of cancer, cardiovascular and respiratory diseases in a higher percentage than problems with alcohol, drugs, hypertension, excess weight or high cholesterol. The enzymes that tobacco smoke induces can increase the risk of cancer by increasing the metabolic activation of carcinogens.

By adopting the public health policies of the last years, the law that prohibited smoking in closed public spaces, determined the important jump of Romania from 12 positions, from the $19^{\text {th }}$ place to the $7^{\text {th }}$ place, in the European classification of tobacco control policies - European Scale Tobacco Control. The direct effects of this policy have reduced the number of hospitalizations associated with diseases exacerbated by passive smoking [1,2]. 


\section{Drug interactions}

Mortality among male and female smokers in the United States is approximately three times higher than among similar people who have never smoked.

Smoking reduces life expectancy and decreases longevity. The life expectancy of smokers is reduced in relation to the number of cigarettes smoked, so that the early cessation of smoking and the long duration of practice will reduce longevity proportionately.

The negative effects of tobacco continue for a long time approximately 10 years and after quitting smoking (fig. 1).

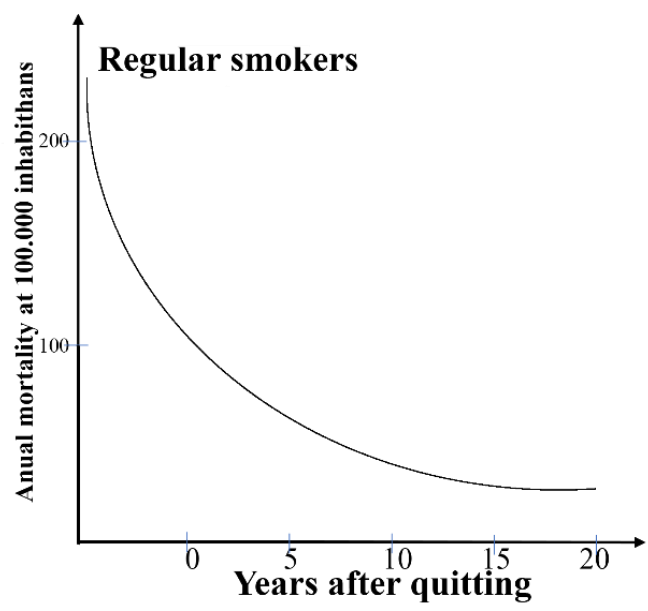

Fig.1 The evolution of mortality in former smokers

In the case of chronic smokers $90-98 \%$ of the inhaled nicotine is retained in the lungs and constitutes together with the tar resulting from burning, one of the main causes of lung cancer growth. In the table 1 is presented the affected organ or system and the type of disease.

Table1. The affected organ or system and the type of disease.

\begin{tabular}{|c|c|}
\hline The affected organ or system & Type of Disease \\
\hline Respiratory system & $\begin{array}{c}\text { lung cancer, laryngeal cancer, reduced respiratory capacity, } \\
\text { pneumonia, chronic bronchitis, pulmonary embolism, asthma }\end{array}$ \\
\hline Cardiovascular device & $\begin{array}{r}\text { tachycardia, cardiac arrhythmia, HTA, poor blood flow, } \\
\text { heart attack, angor pectoris, arteritis, phlebitis }\end{array}$ \\
\hline Brain and Nervous System & cerebral embolism \\
\hline ORL & $\begin{array}{c}\text { laryngitis, pharyngitis, sinusitis, respiratory allergy, } \\
\text { esophageal cancer, neck cancer }\end{array}$ \\
\hline Digestive system & $\begin{array}{r}\text { tongue cancer, gastric cancer, gingivitis, digestive enzymes } \\
\text { destruction, stomach hyperacidity, gastritis, duodenal ulcer, } \\
\text { stomach ulcer, colon cancer }\end{array}$ \\
\hline Excretory system & $\begin{array}{r}\text { kidney cancer, bladder cancer, prostate cancer } \\
\hline\end{array}$ \\
\hline
\end{tabular}

In the case of drug administration in smokers there can be two types of interactions: pharmacodynamics and pharmacokinetics, the latter having consequences on the absorption, distribution, metabolization and elimination of drugs [3]. 
Pharmacodynamic interactions are mainly caused by the effects of nicotine, a major component of cigarette smoke. It enters the body by digestive, respiratory and skin pathways.

Once penetrated into the body, it is rapidly distributed in the blood, and may also cross the bloodbrain barrier. In the table 2 is presented the nicotine content in the different sources.

Table 2. The nicotine content in the different sources

\begin{tabular}{|c|c|}
\hline Source & Nicotine content (mg) \\
\hline Cigarette & $13-32$ \\
\hline cigar & $15-40$ \\
\hline Fag-end & $5-7$ \\
\hline 1 nicotine nasal spray & 0.5 \\
\hline 1 gram of snuff & $12-16$ \\
\hline 1 nicotine patch & $8.3-11$ \\
\hline
\end{tabular}

The metabolism of nicotine occurs in the liver (about 80-90\%). Elimination occurs mainly by the renal route, but can be eliminated mainly by lungs, sweating, milk, saliva, about $10-20 \%$ is eliminated as such, the rest in the form of metabolites (cotinine, as such or hydroxylated). Acidic urine accelerates the elimination, in the case of alkaline urine a resorption occurs in the kidney. It acts at the level of central cholinergic receptors only 7 seconds after inhalation, positively influencing adrenaline activity.

Nicotine, alpha 3 pyridyl-N-methylpyrrolidine, is a toxic pyrrolidinic alkaloid, a characteristic tobacco substance, which imprints both a series of physical properties (color, odor) and a dominant part of the harmful one, being present in tobacco in proportion of $0.7-3.5 \%[2,3]$.

Nicotine produces addiction 6-8 times higher than alcohol, but as high as cocaine. $95-100 \%$ of smokers are addicted. Nicotine, regardless of the route of administration, enters the body through diffusion, inhaled smoke reaches the pulmonary alveoli and all constituents are absorbed. $80 \%$ of nicotine is destroyed in the liver, the remaining $20 \%$ is fixed on nicotinic receptors through which it exerts its multiple effects. In order to measure nicotine dependence the Fagerstrom test is used this test has the advantage that it shows an exact correlation between the test answers and the determinations of plasma and urinary cotinine.

At high doses nicotine is a depolarizing agent of nicotinic receptors. In addition, it results in increased dopamine levels in the brain, due to the inhibition of MAO (monoamine oxidase) responsible for dopamine metabolism. However, it is uncertain whether this action can be attributed to nicotine or other components of cigarette smoke (carcinogenic hydrocarbons). This action on dopamine is similar to that of cocaine and heroin, which is why people smoke: maintaining a high level of dopamine, which causes a feeling of pleasure. Nicotine and its metabolites have been studied for the treatment of diseases such as: Alzheimer's disease, Parkinson's disease. Current research has shown that nicotine alone cannot develop cancer, it does not have mutagenic properties but by increasing the activity of acetylcholine, it leads to blocking apoptosis (programmed death) by which the body destroys certain types of cells, death or mutants, thus developing in cancer cells [4].

Nicotine acts on the sympathetic and parasympathetic vegetative ganglia. Central mechanisms of sympathetic stimulation include activation of chemoreceptors and direct effects on the brainstem and spinal cord. Peripheral mechanisms involve the release of catecholamines from the adrenal glands and vascular nerve endings. These effects are associated with increased levels of circulating catecholamines and, consequently, increased heart rate and blood pressure after smoking. Nicotine also causes coronary and cutaneous vasoconstriction.

In animal studies, nicotine induces the activity of several enzymes, including CYP2E1, CYP2A1/2A2 and CYP2B1/2B2, in the brain, but this effect is not clinically known. It also induces 
CYP2A1/2A 2 in the brainstem and hippocampus and causes inhibition of CYP2A1/2A2 in the cortex and thalamus. Nicotine is metabolized in the liver by CYP2A6.

The polycyclic aromatic hydrocarbons result from the incomplete combustion of all the organic substances in the cigarette being numerous and varied.

Polycyclic aromatic hydrocarbons in cigarette smoke are responsible for the induction of cytochrome P450 (CYP) 1A1, CYP1A2 and possibly CYP2E1, CYP1A1, primarily an extrahepatic enzyme found in the lungs and placenta. The transcription factor, the Aryl hydrocarbon receptor, is activated by a ligand, which regulates the transcription of a wide range of genes, including those responsible for the activity of some enzymes involved in xenobiotic metabolism. These receptors are activated by polynuclear aromatic hydrocarbons, dioxins.

Carbon monoxide inhibits CYP enzymes also selectively inhibits CYP2D6 activity, but does not influence CYP2C and CYP3A activity. The most common effect of smoking on the elimination of drugs in humans is the increase of the rate of biotransformation, in accordance with the induction of drug metabolizing enzymes. [5].

\section{Pharmacodynamic interactions}

In the case of benzodiazepines, smoking decreases the hypnotic and sedative activity, the possible cause being the stimulation of the CNS by nicotine. When smoking cessation the dose should be reduced by $25 \%$ after one week, the possible cause being the increase in plasma levels. In the case of beta-blockers, smoking decreases the antihypertensive and anti-arrhythmic efficacy, the cause being the nice activation due to nicotine. In case of smoking cessation bradycardia and hTA occur, the dose will have to be low. The efficacy of inhaled corticosteroids may be reduced in patients with asthma who smoke. The pulmonary clearance of corticosteroids can be altered by increased airway secretions and permeability. In the case of oral contraceptives, smoking increases the risk of cardiovascular accidents for myocardial infarction, thromboembolism. Oral contraceptives are prescribed to women who smoke more than 15 cigarettes a day. At the same time, in the case of opioid analgesics, smoking decreases the analgesic effect, the doses should be increased in the case of smokers and the cessation of smoking will lead to acute respiratory depression. [5,6]

\section{Pharmacokinetic interactions}

As for caffeine, smoking increases the hepatic clearance by $60-70 \%$. If stopped smoking increases the blood level of caffeine which will lead to severe tremor thus reducing the intake of caffeine by $50 \%$ from food sources. The effect of anticoagulants is decreased by increasing the metabolism that is influenced by smoking, so in smokers the dose of anticoagulant should be increased for the desired effect. Smoking will gradually stop because abrupt discontinuation leads to an increase in INR with a very high risk of bleeding. In the case of warfarin, the dose should be reduced by 14-23\%, after careful monitoring of the INR. Nicotine through pharmacokinetic mechanism and having vasoconstrictive effect, decreases insulin uptake, and through pharmacodynamic mechanism may increase insulin resistance, thus smokers need to increase the dose in order to have a blood glucose between optimal values. In case of smoking cessation, hypoglycaemic seizures may occur, thus monitoring the blood sugar level and decreasing the dose. Theophylline is effected by smoking by increasing metabolism, increasing clearance by $58-100 \%$, decreasing $\mathrm{t} 1 / 2$ by $63 \%$ and increasing volume distribution by $31 \%$. The dose of theophylline should be increased to achieve the desired therapeutic effect. In case of smoking cessation the plasma levels increase (palpitations, nausea), the dose should be decreased (by 25-33\%). Smoking can affect antipsychotic medication, in the case of clozapine the plasma level drops by $18 \%$, in the case of olanzapine the plasma level decreases by $12 \%$ and the plasma level in the case of haloperidol - decreasing by $70 \%$. Antipsychotic doses should be increased in order to have the expected effect. Cessation of smoking results in an increase in plasma level by $72 \%$ in the case of clozapine, an increase in $\mathrm{t} 1 / 2$ by $21 \%$ in olanzapine and an increase in plasma level by $23 \%$ in the case of haloperidol. Doses should be reduced after careful monitoring [2,4,6]. Plasma levels of tricyclic antidepressants (amitriptyline, imipramine, nortriptyline, clomipramine) decrease in smokers, 
so the therapeutic dose should be increased. Stopping smoking leads to increased plasma levels, the dose should be decreased by $10-15 \%$ after one week.

\section{Conclusions}

There are proven connections and interactions in the administration of drugs associated with tobacco use. These are pharmacokinetic and pharmacodynamic in nature and may affect the therapeutic action of the drug and also the patient's health. Thus the smoker must take these aspects into account. Therefore, smokers who undergo drug treatment with a drug that interacts with smoking may require higher doses than non-smokers. And conversely, when smoking cessation, smokers may require a dose reduction of a drug that interacts with smoking. Dose adjustment for smokers and those who quit smoking should be discussed with the doctor and pharmacist for the best health status of the smoker.

\section{References}

[1] Shoshana Zevin, Neal L. Benowitz, Drug Interactions with Tobacco Smoking - An Update, Clin Pharmacokinet, 1999.

[2] Cristea A.N., General pharmacology, Editura Medicala, Bucuresti, 2006.

[3] Nemeroff CB, Kinkead B, Goldstein J. Quetiapine: preclinical studies, pharmacokinetics, drug interactions, and dosing, J Clin Psychiatry, 2002.

[4] Lisa A. Kroon, Drug interactions with smoking, Am J Health-Syst Pharm, 2007.

[5] U.S. Department of Health and Human Services. The Health Consequences of Smoking-50 Years of Progress. A Report of the Surgeon General. Atlanta: U.S. Department of Health and Human Services, Centers for Disease Control and Prevention, National Center for Chronic Disease Prevention and Health Promotion, Office on Smoking and Health, 2014 [accessed 2015 Aug 17].

[6] https://www.ncbi.nlm.nih.gov/pubmed/ 